DOI: https://doi.org/10.33103/uot.ijccce.20.3.2

\title{
Internet of Robotic Monitoring Things (IoRMT)
}

\author{
Marwa Abdullatif Abdelazizi ${ }^{1}$, Ayman D. Salman² \\ ${ }^{1,2}$ Computer Engineering Department, University of Technology, Baghdad, Iraq \\ 120629@student.uotechnology.edu.iq,120008@uotechnology.edu.iq
}

\begin{abstract}
Generally, there is no single aspect of the human life that hasn't been touched through technology. The evolution of technology combines those aspects of technology with modern uses. In this scenario, the electronics of the appliance play a vital role in our life day after day. There are several technologies like IoT Internet of Things, the cloud computing, robotics system, sensors and system on the chips which have revolutionized our technological progress. "IoT" is a vision, which is the biggest development technological technique. There are several platforms of IoT for services developers. A great number of potential platforms choose the most convenient way to perform a specific service. Therefore, developers chose the three of these models which are (blynk), (freeboard) and (dweet.io) to build this system. The proposed system is the Internet of Robotic Monitoring Things (IoRMT), which was employed to monitor the server room in a government security agency, using a set of sensors placed on the car robot. The sensors were connected with ESP8266NodeMCU microcontroller to display the value of sensors in the dashboard of freeboard platform. The car robot was controlled by software joystick using the blynk IoT platform as the mobile android application. The platform IoT dweet.io and platform IoT freeboard.io services and developers from Bug Lab's proved their ability to take data from device and produce it actionable rapidly.
\end{abstract}

Index Terms - IoRMT Internet of Robotics Monitoring Things, Freeboard Platform, IoT Internet of Things, IoRT Internet of Robotic Things, Dweet.io, Blynk and Cloud Robotics.

\section{INTRODUCTION}

System of Robotic has interfered in different socio and economic aspects of a human community. Robot has been used every day to proceed repetitive, boring, dangerous tasks such as packaging, reproduction, monitoring, etc. These applications have been highly effective for achievement in many structures of socio-economical [1]. Technologies of robotic can be integrated with technologies of the network to enhance the up growth of networked robotics. A networked robotic tick to a set of devices robotic is connected by a wireless and wired connecting network to divide the work of sensing, actuating, processing, control among robotics. Networked robotics is limited by resource, data, information and connecting. These limitations lead to many performance degenerations towards cloud robotics [2]. Subsequently, it is integrated to IoT led to Internet of Robotic Things IORT [3]. In fact, it is a new growth of the Internet and can be connecting everything to the internet, anyplace, anytime with any things by using any network or path [4]. Specific definition was selected by IEEE and divided into two environments: First part: for a small environment, IoT is the network to connect Things to the Internet. These Things can be sensing, actuator and programmability, varied from everywhere anytime and anyone [5]. The second part for a large environment, Internet of Things visualizes a selfconfiguring, characterized by or given to adaptation and complex network to connect things to the Internet over the utilized standard connection. The interconnected things are physical or virtual exemplification in the digital world [5]. Evolving IoT in the status of the application may act a heavy mission, fundamentally because of the following challenges: 1) Various languages programming of the devices to be controlled. 2) heterogeneity of the devices Hardware. 3) The constraint of resource 
ingrained to the nodes of IoT. 4) different protocols of communication utilized via the devices [6]. Therefore, two IoT platform were used in this paper, i.e. the Dweet [7] which is a platform used in order to able the data receive from IoT devices over a Humanized web API " HAPI" or API. Dweets can have a loading of up to 2000 characters. Bug Labs is a developed version of Dweet platform, which is a commercial business that is known as Freeboard [8]. Freeboard is the service of the web to be integrated into Dweet which let visualizing values display via many connected devices. Freeboard IoT platform is the maker of strong service named Dweet, i.e. a service of message prepared to IoT devices. Freeboard is an open-source and a free project of the dashboard to be hosted. It is an optional collaboration and simple to merge with different data sources, production fit and elegance design. It is also characterized with easily test and free account. The sources of Data card in the top left panel of configuration is placed to add data of sources to widgets for displaying.

The framework sets a powerful establishment to foster IoT and pick it to the following in-progress notion in the idiom of acknowledged robotics as in "IoRT" Internet of Robotic Things [9]. In specific, IoT architectures are collected from a network to the connection of smart devices, whose pick characteristics of connectivity to the Internet in a combined, interchangeable and processed data [10]. Robotics applications were run to the cloud waterfall into the Platform-as-a-Service (PaaS). The PaaS is an open-source called freeboard that assists robots to offload intensive account by provided that ensured customizable computing in the cloud. The computing environments also permit robots to readily arrival to the RoboEarth learning repository [11].

Cloud robotics is the term to the first mint via James Kuffner in 2010, it points to at all robots which use the cloud for execution code. Merged service of robotics and a cloud of computing, introduces a cloud of networking and cloud of storage. Cloud computing provides faster and high powerful computational ability during large parallel computation and large data storage facilities. It is offered open-source hardware and software. mutual learning ability, during the knowledge of sharing, and knowledge of humans during crowdsourcing.[12]. IoRT is a union of IoT and technologies of robotic taking advantage of IoT and robotic system, IoT concentrates on supporting services of information for tracking and control; the robotics system on making autonomous, action and interaction [13].

\section{LITERATURE SURVEY}

Olimpiya S. et al.,[12], the proposal in this paper was classified into two categories: The development and implementation of system architectures to collaboration cloud computing and robotics. Several architectures of the system to robotics of cloud consist of connection languages and protocols among cloud, database and robots use to control and processing of robot software and architectures. The first specific robotics applications and knowledge of the application like Perception of Computer Vision, Manipulation, Service robotics, Agriculture, Medical and Social. The second discussed the gaps between the research of future and the literature directed to the inclusion of robotic of cloud real-world problems. The drawback in this paper is that the absence of the efficient discussed resource, allocation of task over the cloud and No, control over the robot by the cloud from anywhere and anytime by the "blynk" android mobile application.

Partha P. et al.,2017 [1], produced paper towards the proposed architectural concept of the (IoRT) Internet of Robotic Things that permits robots to share, process, connect and distribute the resources, environmental data, context information and business activities with each other. They proposed a coupling between IoT and robotics together and using the hardware component vehicles, phone, sensors such as weather sensors, industrial sensors, and mobile application home applications. The drawback in this paper is that there is no monitoring system integrated with IoT and robotics. 
Ula K., et al., 2019 [14], produced an environmental pollution system monitoring by using (IoT) .In this paper the system allows the creation of smart environments in which objects interact and cooperate with each other to enable data about air pollutants to be monitored online.

Busra K., et al., 2015 [15], produced a paper towards the cloud platform and discussing the robot and cloud-based robotics. The robot takes advantage of the cloud and network robotics to provide cooperating works. The robot-based cloud can share data, information, and recourse. This paper uses Rapyuta as the platform. The robot has chains in storage, memory, and capacity, therefore, the cloud robotics assistance to overcome these chains utilizes the cloud to connect with the robot together. The drawback in this paper did not use the dweet.io and the freeboard platforms allow the low cost production of the robots because the platforms are free.

Peter M., et al., 2011 [16], this paper deployed Models of the cloud: the cloud of private. The infrastructure of cloud is the provision to a limited use by one organization include multiple consumers such as business units. Private cloud is owned and managed by the organization the blynk cloud. Cloud of Community is the provision to limited use by a specific community of users from organizations to share such as mission, safety requirements, politics Community cloud own, managed by one or more organizations. Cloud of Public infrastructure is provided to utilize by the public. Public cloud is own, managed by a universal, business, organization of government, or group of them used a freeboard. Cloud of Hybrid infrastructure is a group of two or three and more infrastructures of cloud-like private, public and community that stay single structure but are limited together using in this paper this hybrid freeboard cloud and blynk cloud.

\section{RESEARCH PROBLEM}

Taking into consideration all the above which use the IoT, Robot, and cloud, all are employed in our proposed system, but the hardware, software, and design are different according to the new requirements. In this paper, the IoRMT is used to implement the system monitoring by using the car robot platform that consists of microcontroller ESP8266NodeMCU, different IoT platforms and clouds, and cloud computing means to save and enter to the data and programs over the Internet alternatively of your computer's hard drive computer. All these components are used to the monitoring server room in a government security agency. Because this room has a restricted condition on access it, only authorized specific persons have permission to install snd to maintain devices as well as equipment. All actions are monitored via cameras or through the glass window. The robot is used to reduce human effort. So, the accuracy of information about the environment in which the servers must be maintained from high temperatures or humidity, toxic gases and fire.

The proposed robot system is to monitor the environment and notice the temperature, humidity, and fire and sends values to the cloud by dweet.io platform of IoT over thing proxy using API. Then, for displaying the values on the platform called Freeboard display the values of sensors via the cloud on platform freeboard Platform as a service (PaaS) to the cloud computing platform typically contain the operating system and fulfilment environment, database and connection server. The robot can work in any environment and gives better results. The combination array of sensors applied by mobile robots is used to discover their setting and before permit to carefully monitor the environment in real-time moreover, mobile robots are rapid to implement potential to close the implementation into different structures with a modular system of deployment and is able to remove the possibility for human error by playacting easily repeatable tasks, so it becomes better than the integrity of a facility or area. Its disadvantage is the determination on the volume of the capacity that can be carried challenges with wireless connections among the robot and cloud. 


\section{OBJECTIVES AND METHODOLOGY}

\section{A) The hardware components}

The main components of hardware robot car platform are two wheels, dc motor, motor driver L298N board and ESP8266NodeMCU is open-source IoT platform to create devices that can interact with environment. 4 MBytes of ROM (flash), comes with micro USB port, the development board is small size and low cost the ESP8266NodeMCU provides integrated development environment Arduino IDE is cross-platform application write programming language $\mathrm{C}$ and $\mathrm{C}++$ and JAVA. In the last, the WIFI built-in. Figure .1 illustrates ESP8266NodeMCU

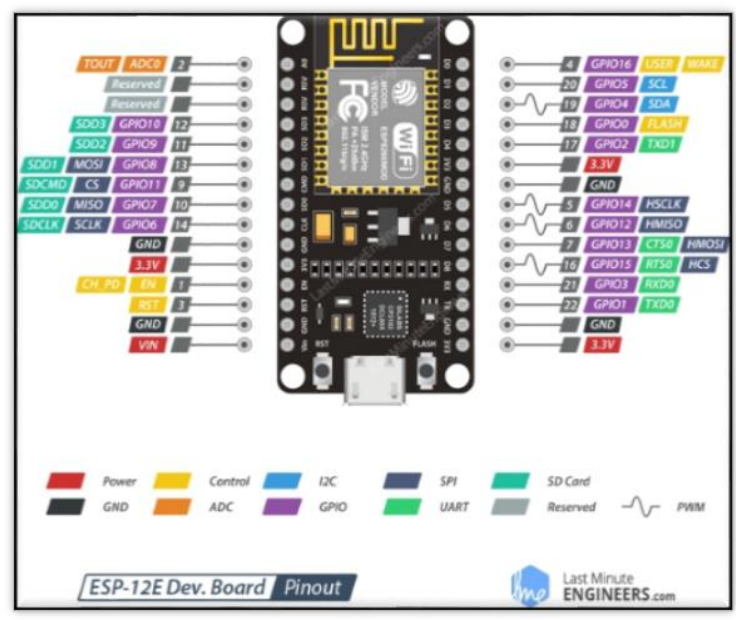

FIGURE 1. ESP8266NODEMCU

Sensors: 1) DHT11 Temperature and Humidity Sensor features are digital signal output. Cheap, Small size, long-term stability, fast response, anti-interference ability, microcontroller, off ering excellent quality, fast response and cost-eff ectiveness. 2) MQ4 sensor message queue module is useful for detecting gases such as co, smoke and LPG and more gases using to monitor the quality of air, alarming to the gas leak, integrity of standard maintenance and environment standards of maintaining in the hospitals. 3) The flame sensor is used to detect fires and light source of wavelength (rang between $760 \mathrm{~nm}-1100 \mathrm{~nm}$.). The sensor is sensitive to infrared radiation, voltage: $3.3 \mathrm{v}-5 \mathrm{v}$, Analog output or Digital Output and Response Time faster. The potentiometer is a simple that set resistance of variable.

In this project, potentiometer values are read in through an 'Analog In' pin. Values are read between 0 and 1023 . The values are then used to control robot speed Use a $50 \mathrm{k} \Omega$ potentiometer. Three wires are Connected to the ESP8266NodeMCU board. First, connect to the ground from one pin of the potentiometer. Second, connect from $3.3 \mathrm{v}$ to the other pins of the potentiometer. Third, connect from the input of the analogue to the centre pins of the potentiometer. Relay Module of two Channel 5v,10A. Relay board of multilateral with inputs of opt isolated. all channel can drive up to $5 \mathrm{~V}$ and $10 \mathrm{~A}$ supply voltage and signals of input size $(3.9 \mathrm{~cm} \times 5.1 \mathrm{~cm})$. motor driverL298N manufactured by ST company, high voltage Dual H-Bridge, Voltage: 7 to $35 \mathrm{~V}$, Peak current: $2 \mathrm{~A}$, temperature $\mathrm{T}=75{ }^{\circ} \mathrm{C}$, board small size $(55 \mathrm{~mm} \times 49 \mathrm{~mm} \times 33 \mathrm{~mm})$, Complete control to movement of dc motors. Integrate two power output (A; B) output power is a bridge configuration, the outputs can drive differential mode or inductive load Depending on the input. Input stage $\ln 1, \ln 2, \ln 3, \ln 4$, enable A, enable B. Figure. 2 illustrates ESP8266NodeMCU Connect to drive motor and two dc motor. And figure. 3 illustrates the main component of the hardware of the prototype. 


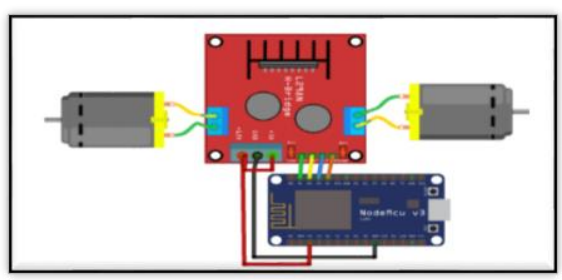

FIGURE.2 ILLUSTRATES NODEMCU CONNECT TO DRIVER MOTOR AND TWO DC MOTOR

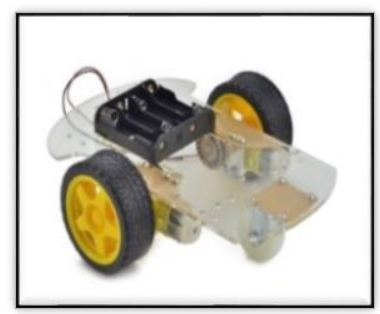

(a)

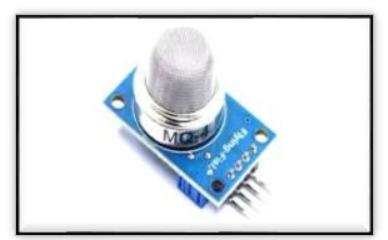

(d)

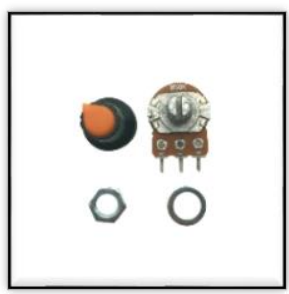

(g)

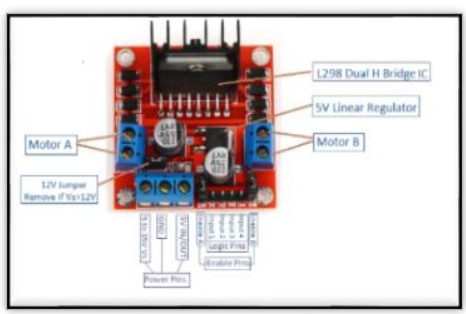

(b)

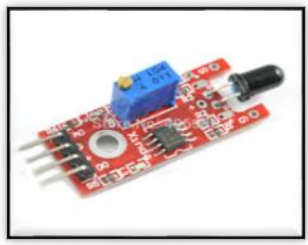

(e)

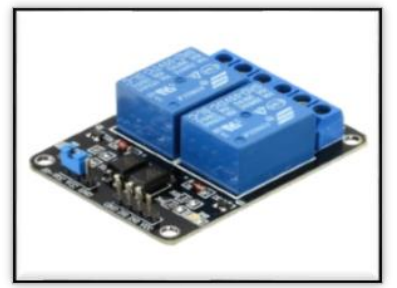

(h)

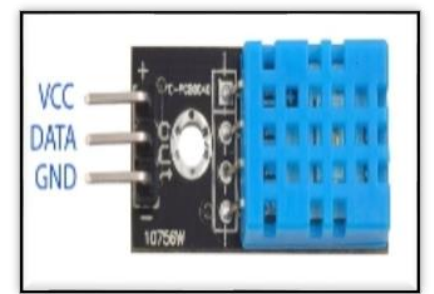

(c)

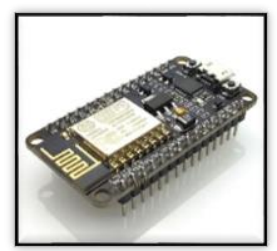

(f)

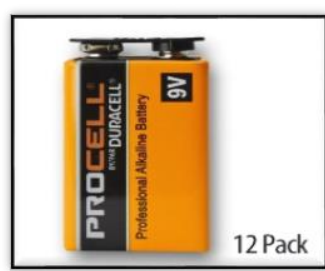

(j)

FIGURE.3 ILLUSTRATES MAIN COMPONENT OF HARDWARE (A) ROBOT CAR (B) MOTOR DRIVERL298N (C) DHT11 SENSOR (D) MQ4 SENSOR (E) FLAME SENSOR (F) NODEMCU (G) POTENTIOMETER (H)RELAY MODULE (J)BATTERY.

\section{(B) Software:}

1) Arduino IDE programmed using programming language $C$ and $C++$ and JAVA.

2) Blynk cloud platform, so it can control the robot by software joystick from anywhere in the world, the control of robot is through joystick software instead of joystick hardware, as well to reduce the cost and ease of installation blynk application on the mobile to use easily software joystick. This cloud platform was chosen because management Connection: Library of the blynk can connect to any hardware over GSM, Wi-Fi, Ethernet, or 2G, 3G, LTE.

Designed for IoT: control equipment remotely, manage multiple devices, set alarm and rules. API Flexible: comprehensive hardware-cloud-app API with language python $\mathrm{C}, \mathrm{C}++, \mathrm{HTML}$. Private servers and public: the cloud of the blynk is open source, can run by us, in the cloud environment like iCloud, Amazon and can host private on a local machine. Reliable and fast: Server of blynk is realtime and deployable in minutes, can manage billions of requests from devices. low cost and Timeeffective .3) freeboard platform is a (PaaS) platform-as-a-service is one of three fundamental Cloud computing provides a framework to robotics helped human to interface robot and monitoring sensors 
and display the value to the dashboard. Other fundamental of cloud computing SaaS Software as a Service, IaaS Infrastructure as a Service [17].

\section{IORMT APPROACH}

The proposed system was designed as to monitor the room of control server because this room contains important data and does not allow any human access to them only when the installation and maintenance of these servers. Figure .4 illustrates the system prototype, so this robot is designed to monitor the environment for this room. Control the robot through the cloud from anywhere and anytime in the world by connecting the robot to the cloud. So, controlling the robot through a private cloud by the application of a mobile is called Blynk. Figure .5 illustrates how to connect car robot to driver motor and dc motor .with software upload to ESP8266NodeMCU, we need to install the Blynk library and ESP Wi-Fi library, this application allows us to manufacture your Joystick by smartphone and thus reduced the price and physical parts instead of using hardware with Joystick software, this allows anyone who connects to the mobile application and the mobile works as a controller of this robot. Robot car and contains a microcontroller to control the robot from anywhere in the world and real-time with the use of 2 Channel Relay Module 5v,10A between driver motor L298N and DC motor because the instant ESP8266NodeMCU first takes the power of each pin becomes high. This time is enough to process the sensors, the distribution, and identification of the pins. ESP8266NodeMCU as a brain of the robot to control and linked with the cloud.
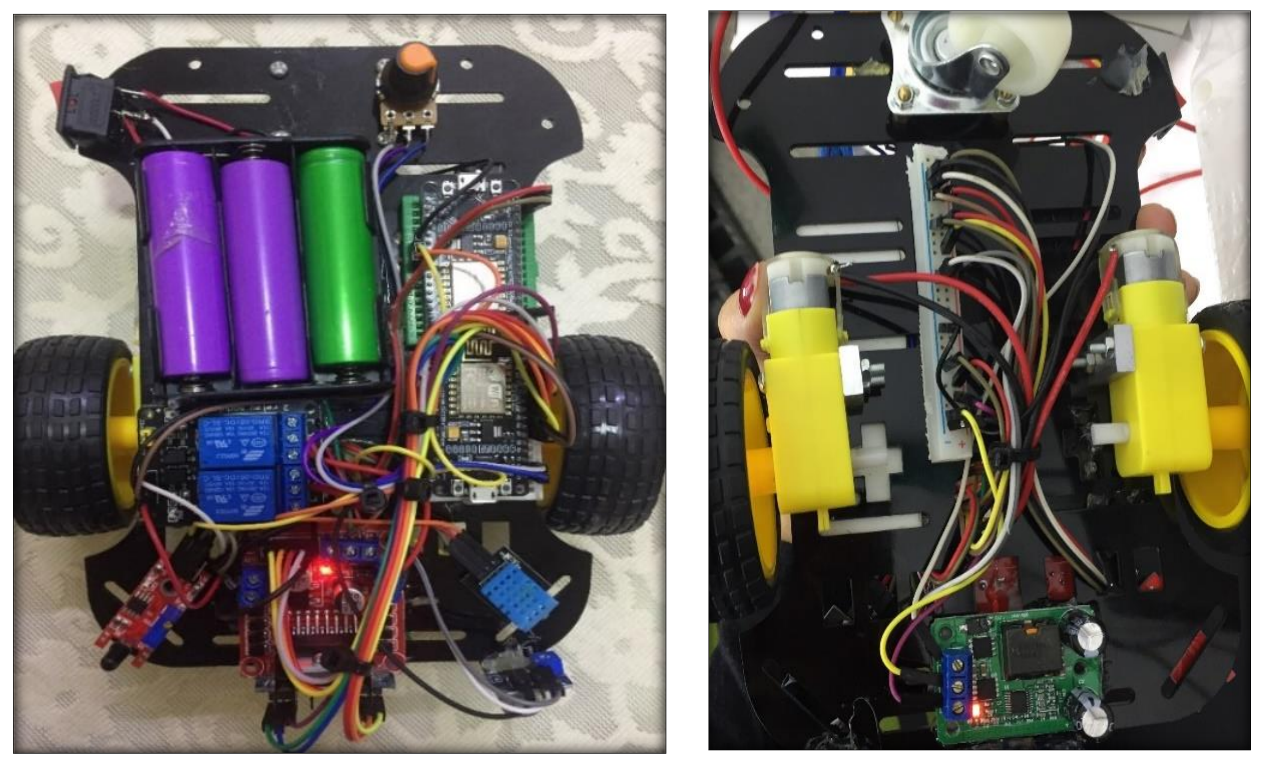

FIGURE .4 ILLUSTRATES THE SYSTEM PROTOTYPE 


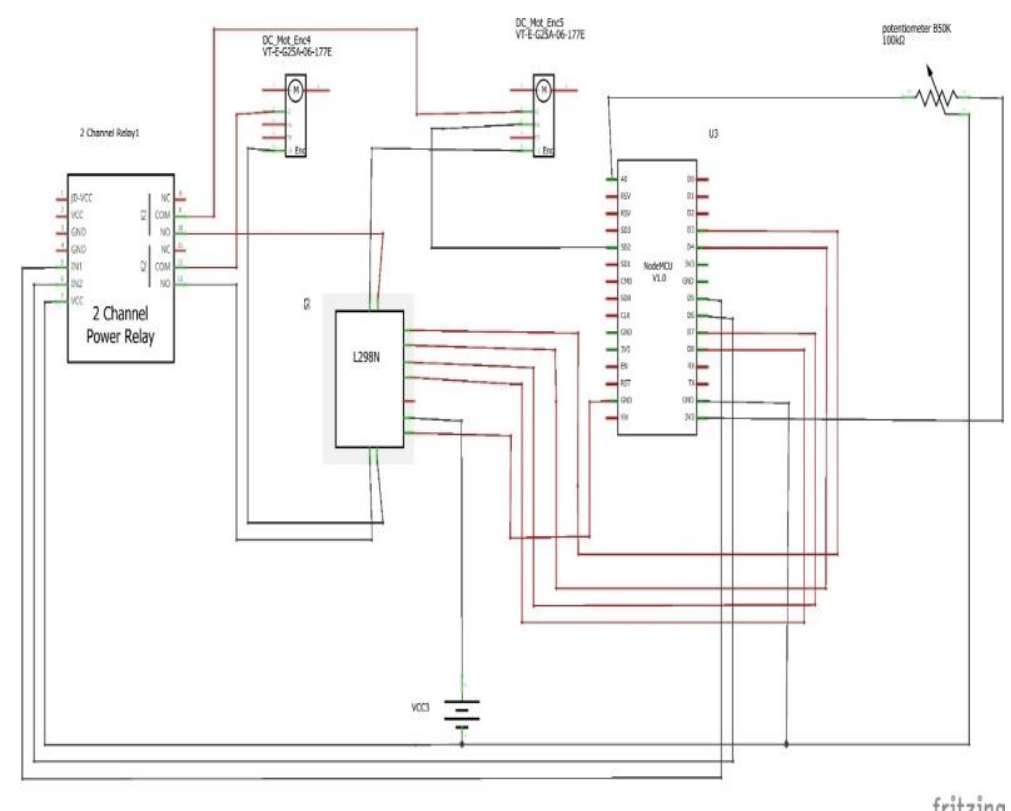

FIGURE.5 ILLUSTRATES BLOCK DIAGRAM SHOW CONNECT CAR ROBOT WITH DRIVER MOTOR, DC MOTOR, RELAY MODULE, VCC, POTENTIOMETER TO NODEMCU

Second microcontroller ESP8266NodeMCU connects with sensors for this robot. Figure.6 illustrates how to connect the ESP8266NodeMCU with sensors and Buzz. In case of high temperature, humidity determines the presence of fire, any type of toxic gassing such as co, smoke, and LPG gives an order to

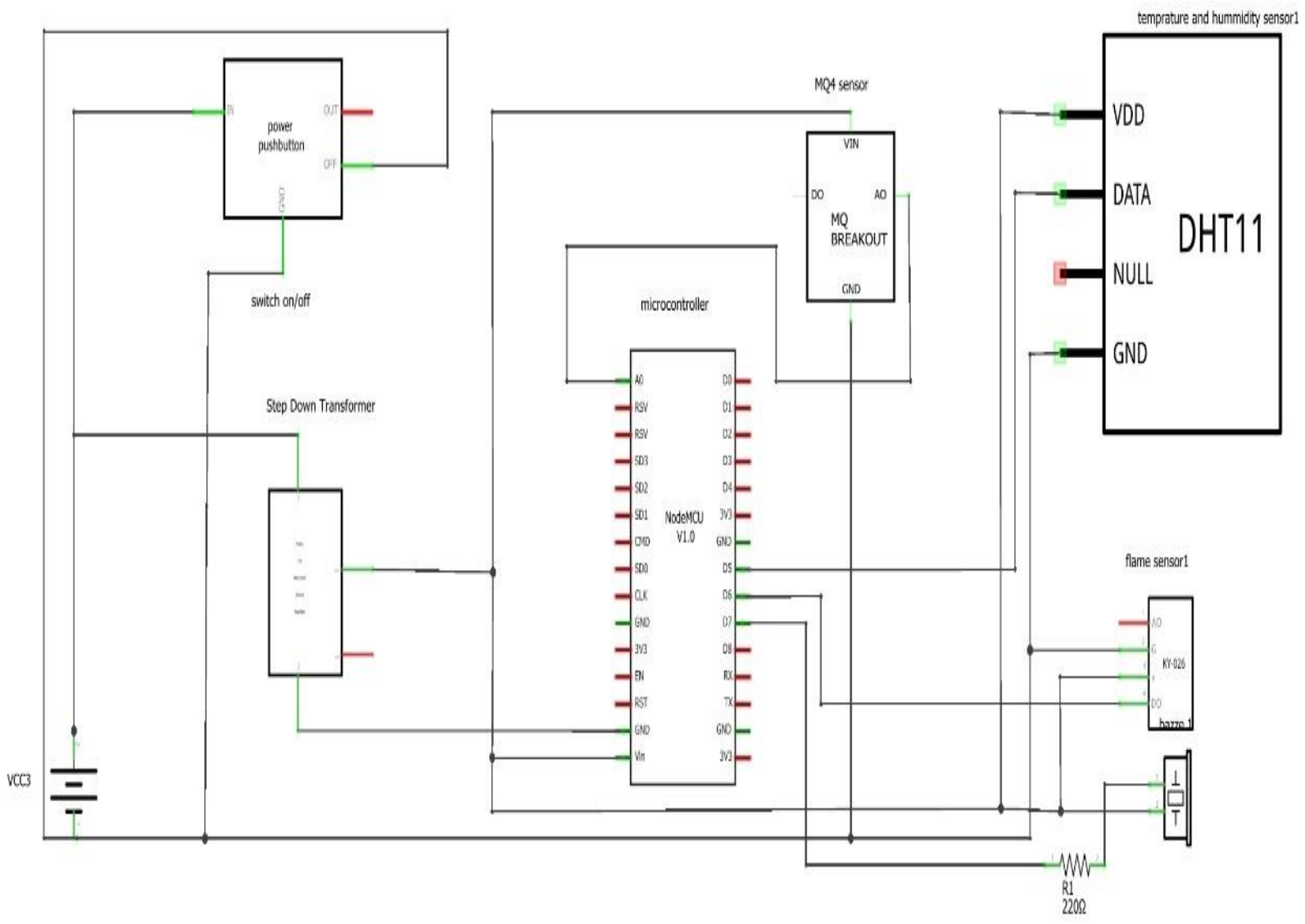

FIGURE.6 BLOCK DIAGRAM EXPLAIN HOW TO CONNECT THE NODEMCU WITH SENSORS AND 
measure the server room environment. Then display values through a platform called Free Board and so this platform provides compatibility between robot and web applications because the web contains one processor and the robot may contain more than one processor, as well as protocols used in robot differ from the one used in the web and used for platform compatibility, which is a public cloud, open sources, speed calculations and distribution of tasks between robot and cloud.

It was used two Microcontrollers (ESP8266NodeMCU) because of the private cloud libraries, namely the blynk not compatible with the public cloud platform freeboard libraries .And the freeboard does not have the joystick, therefore, it was used the blynk for controlling the robot. The distribution of work was on both microcontrollers as well as to achieve the speed of robot response to the cloud. The operation of the robot is through the mobile application and making your mobile as a joystick to control the robot in less than a mall second. thus, achieve a response during real-time. Blynk is a platform that uses with IOS and android application to control ESP8266NodeMCU over the internet Figure.7 illustrates how the configuration of blynk application in the smartphone. It has a graphical interface that is used to build projects by dragging the widgets.
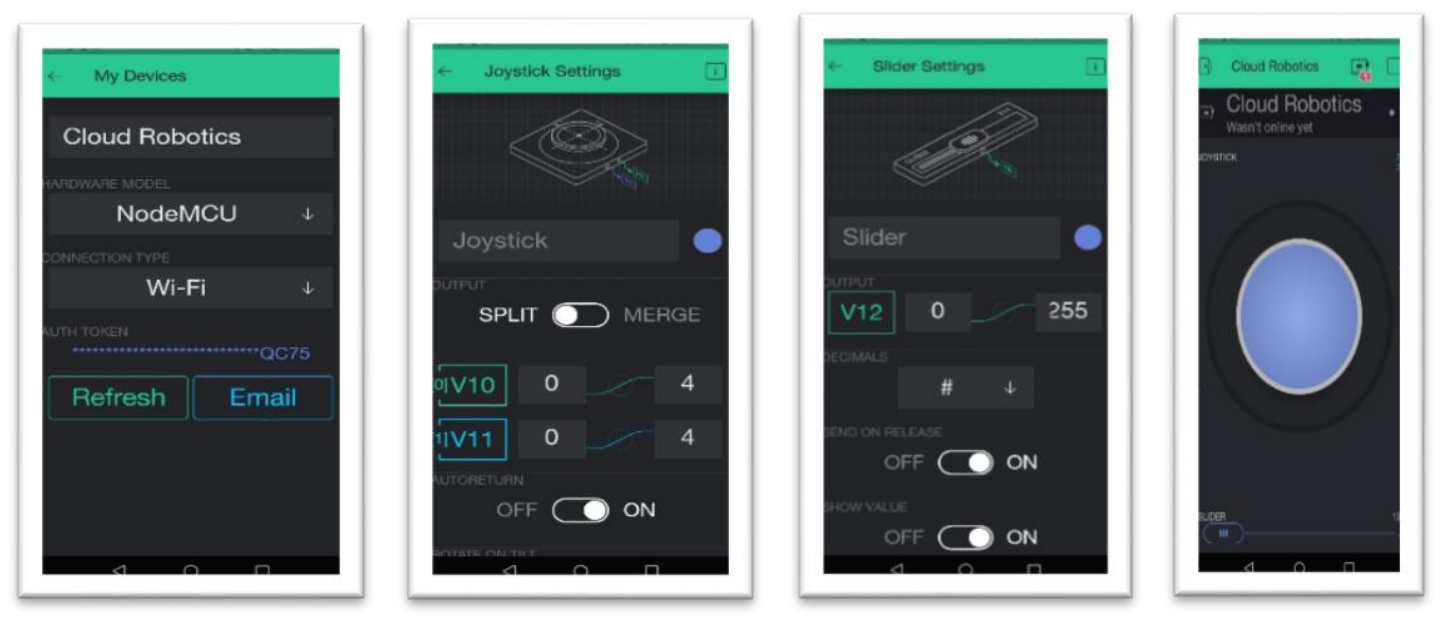

FIGURE.7 ILLUSTRATES HOW CONFIGURATION OF BLYNK APPLICATION

Freeboard adopts a minimalistic approach for its interface and displays the graphics. This approach produces the service truly easy to use. Freeboard is closely integrated with Dweet. Figure. 8 illustrates the results of sensors on Dweet IoT platform. 


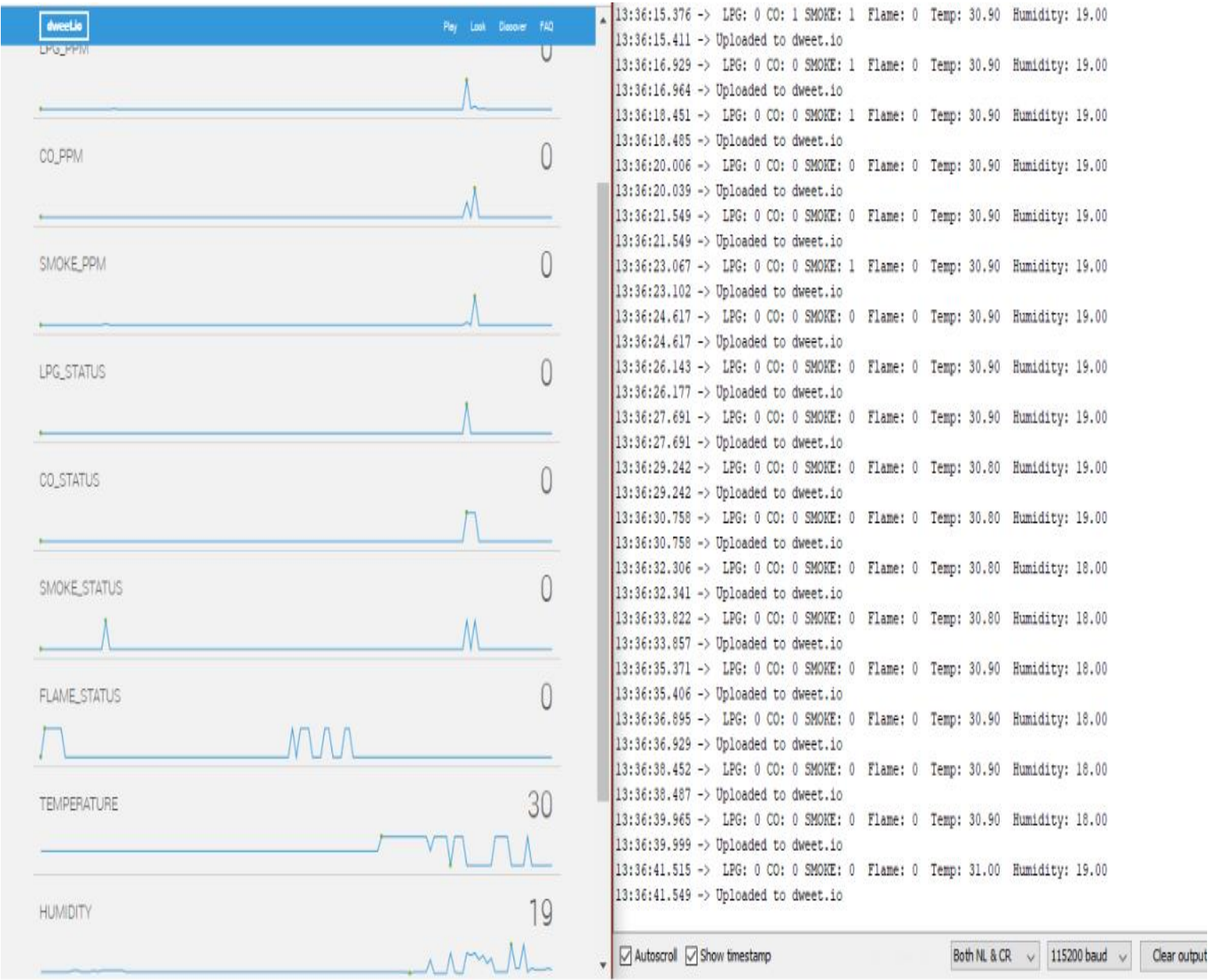

FIGURE.8 ILLUSTRATES RESULTS OF SENSORS ON DWEET IOT platform

The dashboard senses automatically for freeboard platform IoT, see the results in Figure.9
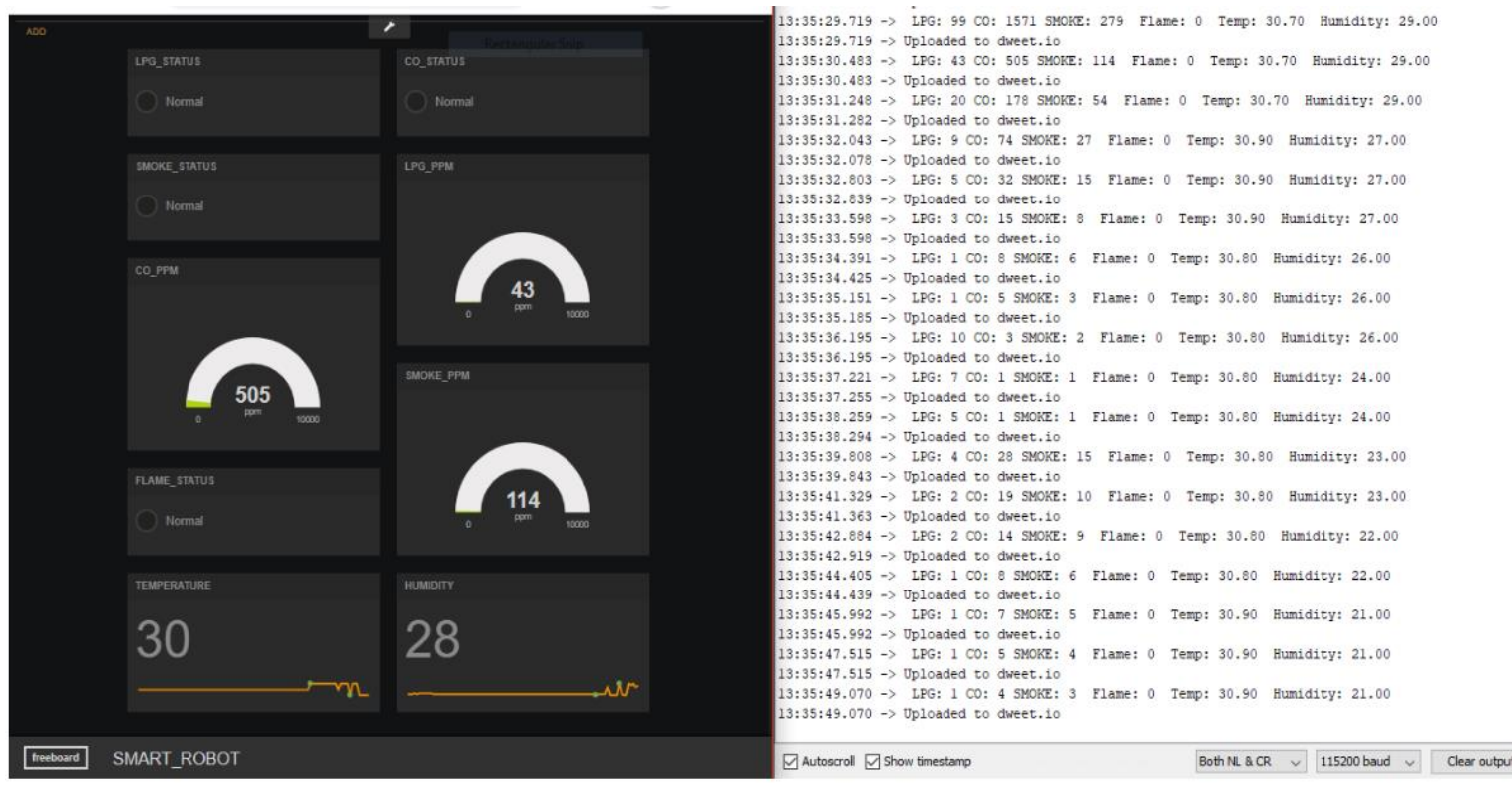

FIGURE.9 ILLUSTRATES DASHBOARD OF SENSORS IN THE FREEBOARD PLATFORM 
The figure.10 illustrates the structure of IORMT, The summary of each module is as follows: 1) User. 2) IORMT_cloud: node for communicating with a cloud (private and public). 3) IORMT_UR: node for communicating with user and cloud by a private cloud named blynk. 4) IORMT_TS: node for planning service tasks performed by the robot. 5) IORMT_B: Connect to the private cloud(blynk). 6) IORMT_SD: node for controlling sensors such as DHT11, MQ4, and flame sensors.7) IORMT_DV: Display value to cloud platform named freeboard. 8) IORMT_RP: nodes for planning robot motions by Software joystick 9) IORMT_RC node for executing robot motions.

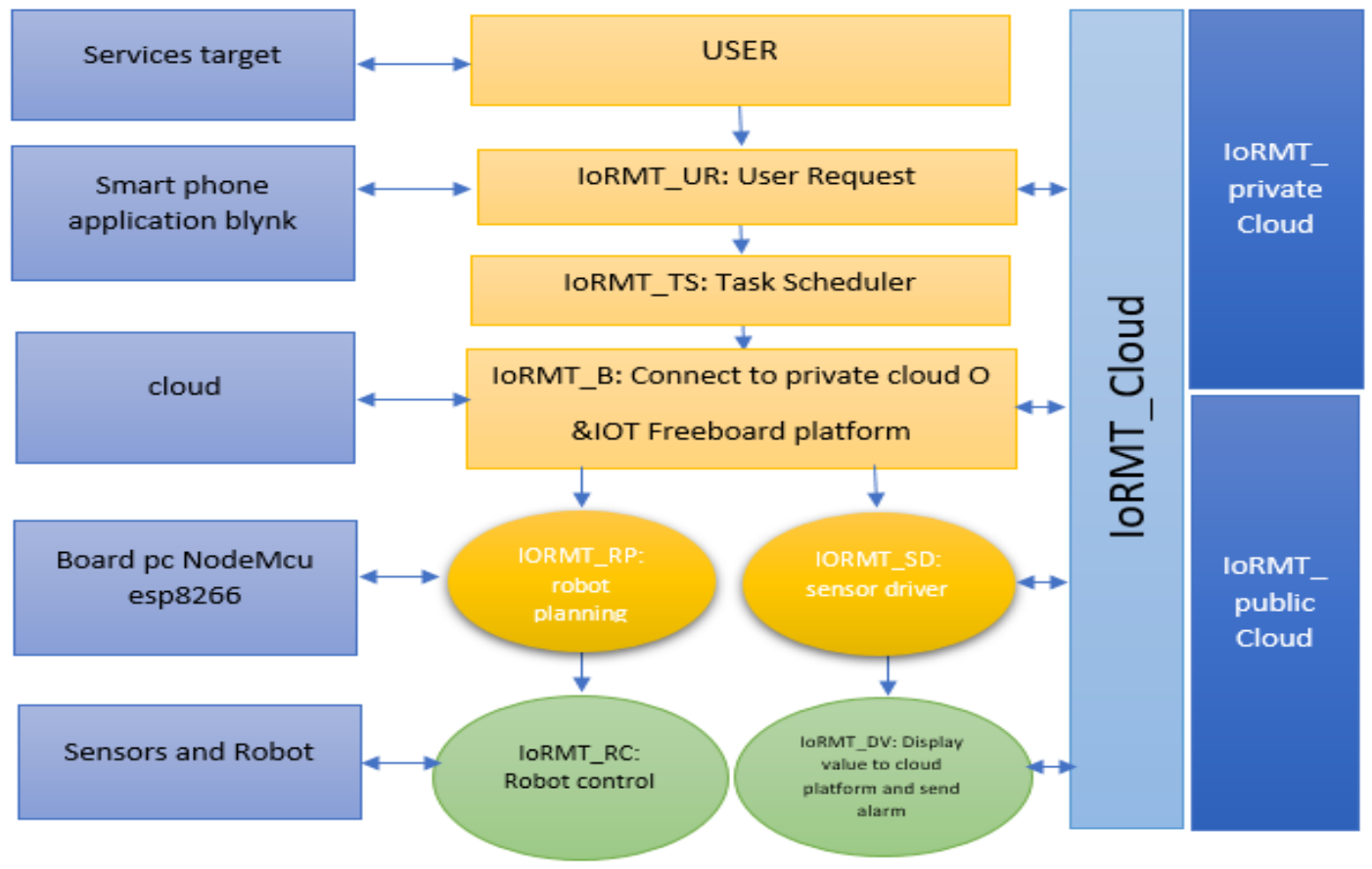

FIGURE 10 ILLUSTRATES STRUCTURE IF IORMT

A cloud setup architecture for extensive nonautonomous robots has been proposed Figure.11 illustrates the architecture of the cloud platform. The architecture consists of three subsystems: (1) Subsystem of Middleware: Robotics language, User-friendly and Compatible with cloud (2) Subsystem of Background Tasks: Programming language and software Arduino IDE uses language C, C++and java. Libraries like Esp8266WiFi, Blynk, freeboard and dweet.io. The setting for batch processing included software packages. (3) The subsystem of Control: Computation, Storage and Networking. which is the intelligence of the platform. So that, the subsystems are horizontal of requested, and vertically of processer of the networked, storage and computation. 


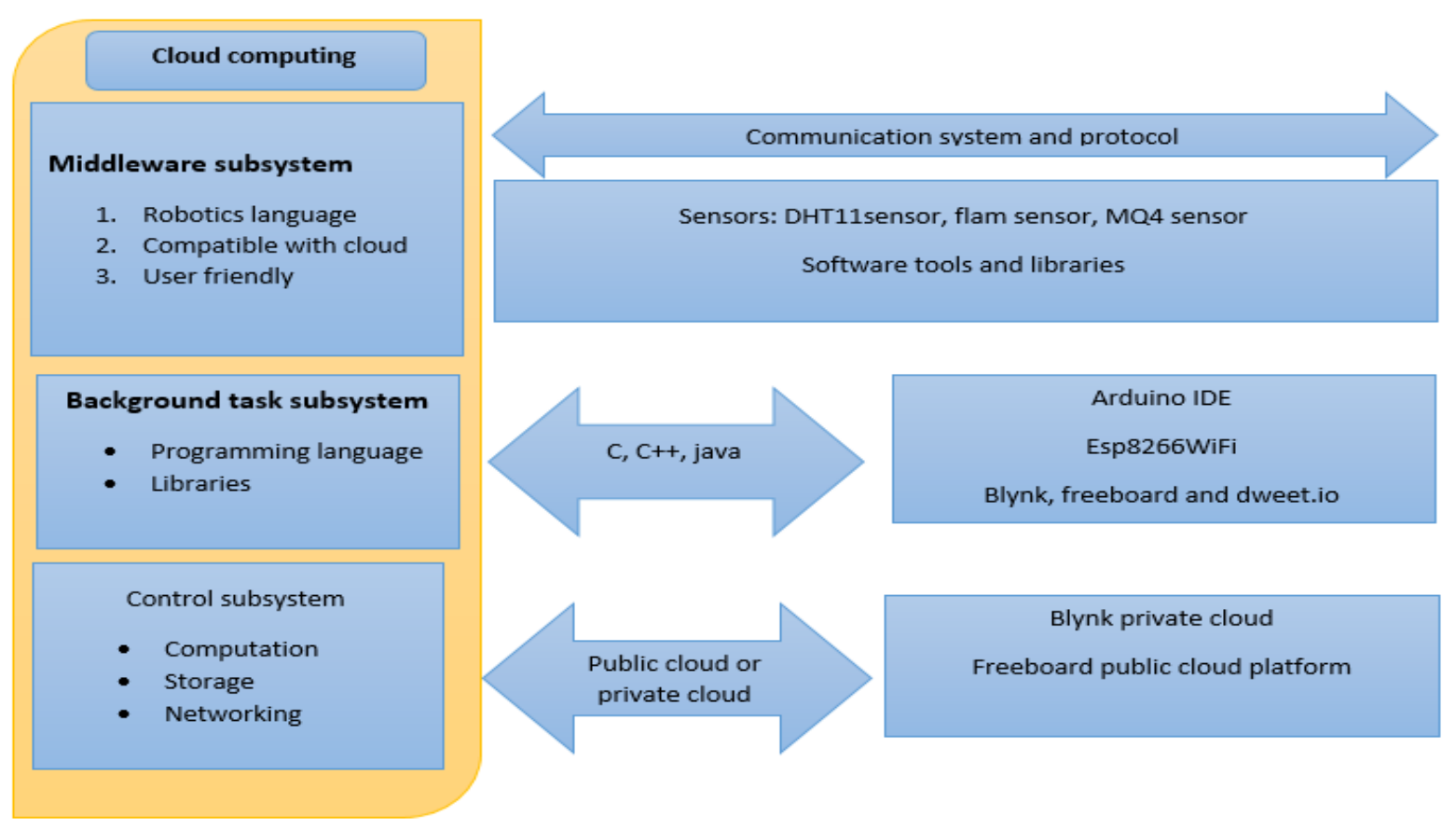

FIGURE.11 ILLUSTRATES THE ARCHITECTURE OF CLOUD PLATFORM [2]

A flowchart will be illustrated in Figure 12. The part of the software that exemplifies the algorithm of the sensors system in a step of the working of this system can appear in the following points:

1) The system starts with push-button on/off.

2) Check if it is controlling the robot through joystick, if yes control by private cloud named blynk.io then directional control by joystick, then control the speed of the robot car by blynk IoT platform.

3) The system read the three value of sensors then send these values to dweet.io IoT Platform for transposition and then display value into the dashboard of freeboard IoT platform to be monitored by the employ.

4) Values of sensor testing start, gas sensor (MQ4) value at first. If gas is detected, the system will send a real-time buzz alarm to the user.

5) Then, test flame sensor values of the fire, if the fire is detected, the system will send a real-time buzz alarm to the user.

6) Then, test DHT11 sensor values of the temperature and humidity, if temperature value is above $26^{\circ} \mathrm{C}$ or humidity is above 30 , the system will send a real-time the buzz alarm to the user. 


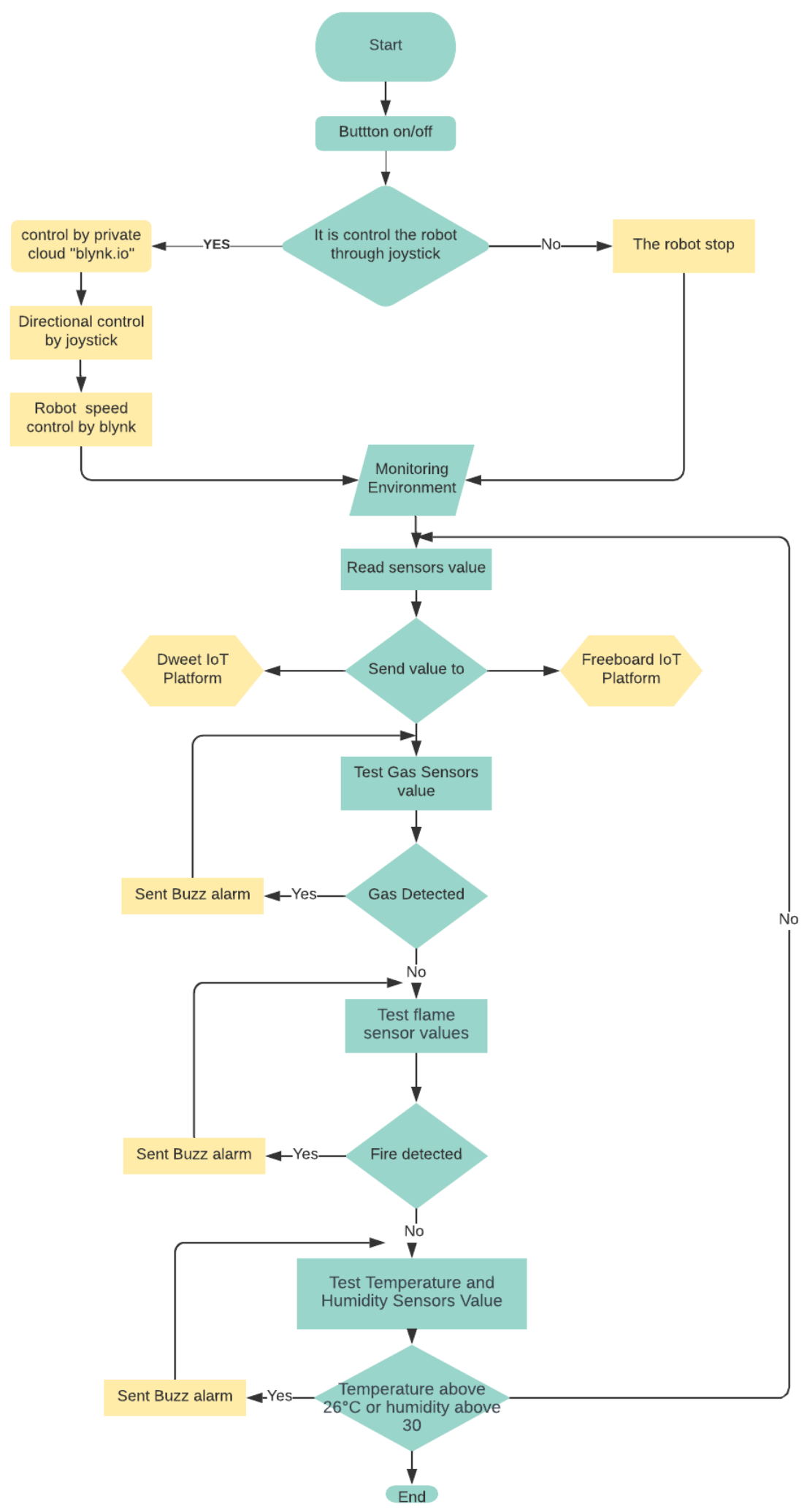

FIGURE.12 THE FLOWCHART OF THE SYSTEM 


\section{VI.CONCLUSION}

This paper presents the robot monitoring architecture which is based on IoT platforms and uses (dweet.io, freeboard.io and blynk.io). The robot is designed with three IoT platforms for monitoring server room in a government security agency. The constructed system is named IoRMT. The system consists of sensors to measure temperature, humidity, gases and detect fire in order to protect the servers because it is very important evidence. Thus, the aim was to keep this server safe from the changeable environment, to increase reliability, and to reduce the complex calculations. The results show that the system can connect the robot to cloud and the results of the sensors on the platform. The research has surfed into how the IoT systems is merged through robotics of the cloud. How could be benefit from operations of the robotic such as the sensing, the manipulation, the monitoring etc. This paper discusses also the ways in which the robots could act as the extension to the devices in IoT. Moreover, it was easy to implement the dashboard because of the ease of utilizing the solution and the integration between Dweet and freeboard.

\section{FUTURE WORK}

Autonomous and non-autonomous are integrated into future work. That can add sensor IR to convert the robot control by human "non-autonomous" to self-organize "autonomous" and add new IoT platform pushbullet.

\section{REFERENCES}

[1] Partha P., "Internet of Robotic Things: Concept, Technologies, and Challenges," IEEE ACSSES, vol. 4, no. 2016, pp. 9489-9500, 2016.

[2] Seyed A., Nicolas G., Nicholas G., Karthikpai H., Abhijith R ., Paul R., Mo J., " Cloud robotics: A software architecture: For heterogeneous large-scale autonomous robots, IEEE, vol. 6, no. 2016, pp. 978-1-5090-1895-6, 2016.

[3] Koji K., Shuichi N., Norihiro H., and Miki S., "Cloud networked robotics," Network, IEEE, vol. 26, pp. 28-34, 2012

[4] Ovidiu V., Peter F., "Internet of things _from research and Innovation to market deployment"river publishers series in communications, p. [56], 2014.

[5] James. K, "Cloud-enabled robots," IEEE-RAS International Conference on Humanoid Robotics, Nashville, TN, 2010.

[6] Dr.Aymen Dawood Salman, Dr. Ghaidaa Muttashar Abdulsaheb, and Ula Khudeer Abbas, " Air Quality Management System in a Smart House Based on IoT", Journal of International Conference on Research and Modern Innovations in Engineering \& Technology, ICRMIET, 2019.

[7] Dweet—Data Sharing for IoT. Available online: https://dweet.io/ (accessed on 2 November 2019).

[8] Freeboard-Dashboards for the Internet of Things. Available online: https://freeboard.io/ (accessed on 2 November 2019).

[9] Anand N., Ranbir S., Amandeep N., "Internet of Robotic Things: Driving Intelligent Robotics of Future- Concept, Architecture, Applications and Technologies, " IEEE ACSSES, vol. 4, no. 2018, 2018.

[10] Kumar Y., Md S. A., and Ahmed A., "An Application-Driven Modular IoT Architecture," Hindawi Wireless Communications and Mobile Computing, vol. 2017, Article ID 1350929, pp. 1-17, 2017.

[11] Dominique H., Mohanarajah G., Markus W., and Raffaello D., "Rapyuta: The RoboEarth Cloud Engine,". Future Gener Compute, vol. 74, pp. 337-348, 2017.

[12] Olimpiya S. and Prithviraj D., "A Comprehensive Survey of Recent Trends in Cloud Robotics Architectures and Applications," Robotics MDPI, vol. 3, no. 5, pp. 9489-9500, 2018.

[13] Pieter S., Mauro D.and Alessandro S., "The Internet of Robotic Things: A review of concept, added value and applications, " International Journal of Advanced Robotic Systems, pp. 1-11, 12 October 2017.

[14] Ula Khudeer Abbas, Dr.Aymen Dawood Salman and Dr. Ghaidaa Muttashar Abdulsaheb, "Smart city Management System Using Wireless Sensor Network", Submitted to Journal of Communications and Control \& Systems Engineering (IJCCCE), 2019.

[15] Busra K., "CLOUD ROBOTICS PLATFORMS," Yalova University Yalova, Turkey, pp. 27-33, 12 October 2014.

[16] Peter M., Timothy G.," The NIST Definition of Cloud Computing, " National Institute of Standards and Technology, Special Publication 800145, 7 pages, (September 2011).

[17] Blynk_IoT platform and private cloud Available online: https://blynk.io/ (accessed on 2 November 2019). 